\title{
Cela s'arrête là
}

\author{
Armelle Chitrit
}

In the tradition of the ancient Greek chorus, Armelle Chitrit's "It stops here" is a poem in two voices. It was written for the film "De l'ombre à la lumière," a documentary by Lise Bonenfant which allows women who have suffered conjugal violence to take control of their lives through artistic expression. The first part of the poem stages the recognition of violence; the second voices anger and resistance; and the third offers a message of hope for women who live with violence. Although the poem does not suppress the terms of violence, it shows that through language and symbol women can refuse sacrifice and break the cycle by which violence returns again and again to haunt them. In this way, the poem generates a sense of solidarity and an opening onto the world.

\section{I}

En creux de cette histoire il y en a beaucoup d'autres qui ne s'écrivent pas

Et dire que ça pourrait s'arrêter là

Notre mémoire est un désert où la question trace Et dire que ça pourrait s'arrêter là une absence de fin

La route donc se poursuit toujours. Exilée du lieu-dit. Attelée au seul souffle.

Et dire que ça pourrait s'arrêter là

Et de la fleur au fruit coûte que coûte

II

La nuit est froide de silence Et dire que ça pourrait s'arrêter là Des astres sont tombés de haut quand on y pense.

Ils sont chassés parmi les mots. 
Ah! Qu'un front sorte de la blessure! mais les mots fuient entre mes hanches, comme des fantômes sans nourriture, Et dire que ça pourrait s'arrêter là au détour de leurs boucles blanches,

Qu'un front sorte de la blessure! $\mathrm{O}$ mon amour, mon tout petit Que d'un fruit le souffle de vie.

\section{O-U-I.}

J'attends le midi du silence

Cela pourrait s'arrêter là.

Je veille l'éveil enseveli.

\section{III}

Les consonnes des maisons de chairs s'érigent au-dessus de nos cris Je veux partager ma prière d'un grand soleil en plein midi

Les voyelles pleines de lumière ouvrent au ciel leurs orifices, mon fier amour, mon tout petit, mes âmes sœurs, mes hommes frères refusent enfin le sacrifice.

Cela ne s'arrêtera pas.

Cela ne s'arrêtera pas.

Cela pourrait s'arrêter là. 